\title{
Preparation and evaluation of nanoparticles for ocular delivery of diclofenac sodium
}

\begin{abstract}
Background and objective: Ocular drug delivery research is challenging due to the presence of anatomical and physiological barriers. The ophthalmic preparation of diclofenac sodium (DC) for relieving ocular inflammation is presently available in the market only as an eye drop solution with low ocular bioavailability. The goal of our study was to prepare and evaluate an eye drop formulation, containing a nanocarrier, chondroitin sulfate (CS)-chitosan ( $\mathrm{CH}$ )-nanoparticles (NPs) (CS/ CH-NPs) is being evaluated for improving the bioavailability of diclofenac sodium into the eye.

Methods: Formulation was developed and optimized for three different variables (chondroitin sulfate, chitosan, and initial drug concentration). The developed formula was evaluated by various in-vitro parameters such as particles' size, entrapment efficacy, zeta potential, and in vitro release profile.
\end{abstract}

Results: The particles' size, entrapment efficacy, and zeta potential of the best formulation were found to be $251.1 \pm 15.12 \mathrm{~nm}, 73.2 \pm 5.3 \%$, and $38.89 \pm 5.05 \mathrm{mV}$, respectively. Furthermore, the best formulation showed a biphasic release profile, initial burst release in the first hour, followed by sustained release within 24 hours.

Conclusion: Promising results of in-vitro studies indicated that CS-CH-NPs could be a potential substitute for improved ocular delivery of diclofenac sodium.

Keywords: Chondroitin sulfate (CS); Chitosan (CH); Nanoparticles (NPs); Diclofenac sodium (DS).

\section{Introduction}

Ophthalmic drops are the most commonly used formulations due to the ease of administration and patient compliance. However, the bioavailability of the active substance is less than $5 \%$ due to anatomical and physiological limitations such as lacrimal turnover, blinking reflex, nasolacrimal drainage, or ocular barriers. ${ }^{1}$ The ocular drug bioavailability can be improved by either prolonging retention time in the cul-de-sac or by increasing the ocular permeability. ${ }^{2}$ The main purpose in the development of ophthalmic formulations is to obtain an optimal concentration of the active substance and to maintain it for a longer period of time, thus reducing the frequency of administration. ${ }^{3}$ To overcome the inconvenience of topical ophthalmic preparations, researchers have approached two strategies. First by increasing corneal residence time using viscosity enhancers, mucoadhesive agents, and in situ gels. Second, by increasing corneal permeability using penetration enhancers, prodrugs and colloidal systems such as nanoparticles and liposomes. ${ }^{4}$ In this review, we highlight some nanoparticles (NPs) appeared as an elegant approach to enhance the ocular bioavailability of topically instilled drugs into the eye cavities and preserving the ease of delivery. These systems offer inimitable features, such as non-irritancy, prolonged residence time, sustained effect, high corneal permeation, and reduced dosing frequency, and thus patient

${ }^{1}$ Department of Pharmaceutics, College of Pharmacy, Hawler Medical University, Erbil, I raq.

* Correspondence: naz.ibrahim@hmu.edu.krd 
compliance and reduced systemic side effects. ${ }^{5,6}$ Chitosan $(\mathrm{CH})$ is the most widely explored polymeric nanoparticle for ocular delivery and the most investigated system to enhance corneal and conjunctional penetration. 7 Chitosan is poly $[\beta-(1-4)-$ linked-2-amino-2-deoxy-dglucose]; it is a natural cationic polysaccharide obtained by chitin deacetylation. It has been extensively investigated in the past decades for its application potentialities in the pharmaceutical field due to its unique characteristics, such as non-toxicity, biocompatibility, and biodegradability, as well as its favorable muco adhesiveness and biomembrane permeability. ${ }^{9,10}$ Chondroitin sulfate (CS) is found in bone, cartilage, and connective tissue; it composed of acetyl galactosamine and glucuronic acid residues, alternately linked to each other by the $\beta 1-4$ and $\beta 1-3$ bonds, respectively. CS is an anionic mucopolysaccharide, which is able to form ionic complexes with positively charged substance. ${ }^{11}$ Chitosan- Chondroitin sulfate NPs formulation is based on electrostatic interaction between the protonated amine group of chito-san and negatively charged group of Chondroitin sulfate (CS) or an anionic polymer. ${ }^{12}$ Complexation of $\mathrm{CH}$ with anionic CS has been reported to decrease its solubility. This property is useful in sustaining the drug release. The interaction of the polymer with an oppositely charged polymer results in an exchange of counterions, and it is accompanied by an increase in entropy. ${ }^{13,14}$ Diclofenac sodium (DC) is one of the nonsteroidal antiinflammatory drugs approved by the US Food and Drug Administration for ophthalmic use as an eye drop. It can inhibit inflammatory reactions in the eye through inhibiting prostaglandin synthesis owing to suppressing Cyclooxygenase enzyme. ${ }^{15}$ The ophthalmic preparation of DC for relieving ocular inflammationis available in the drug market only as an eye drop. $^{16}$ It has been extensively used in patients suffering from postoperative ocular inflammation and pain associated with cornea defects after surgery with a frequency of three to six times daily. Therefore, a minimized frequency of administration to one to two times a day will be beneficial for patients. ${ }^{17,18}$ This study aimed to prepare and evaluate an eye drop formulation containing nanocarrier of chondroitin sulfate (CS), chitosan $(\mathrm{CH})$ nanoparticles (NPs) $(\mathrm{CS} / \mathrm{CH}$ -NPs) for improving bioavailability and minimizing the frequency of administration of diclofenac sodium.

\section{Methods}

\section{Design, setting and time of the study}

This experimental research study was carried out in the College of Pharmacy, Hawler Medical University from $1^{\text {st }}$ of March 2017 to $30^{\text {th }}$ of August 2017.

\section{Materials}

Chitosan $(\mathrm{CH})$, and chondroitin sulfate (CS) were procured from Sigma Aldrich. Diclofenac sodium was provided by Jubilant Organosys Limited, India. Methanol, sodium bicarbonate, sodium chloridedihydrate, and calcium chloride were purchased from S.D. Fine Ltd, India.

\section{Methods:}

\section{Preparation of buffer solutions}

Simulated tear fluids were prepared according to a procedure as follows: Sodium bicarbonate $(200 \mathrm{mg})$, sodium chloride $(670 \mathrm{mg})$ calcium chloride di-hydrated $(8 \mathrm{mg}$ ) were dissolved in purified water and made the volume up to $100 \mathrm{ml}$ then $\mathrm{pH}$ adjusted to $7.4 .^{19}$

Preparation of chondroitin sulfate-chitosan nanoparticles (CS-CHNPs)

A stock solution of $\mathrm{CH}(1 \% \mathrm{w} / \mathrm{v})$ was prepared by dispersing it into $2 \% \mathrm{v} / \mathrm{v}$ of acetic acid solution. Similarly, $1 \%$ stock solution of CS was prepared by dissolving it into distilled water. Both solutions were serially diluted thereafter $\mathrm{pH}$ of $\mathrm{CH}$ solution was adjusted to 5 followed by filtration before formulation development. Formulations were developed by ionic gelation method. ${ }^{20}$ An equal volume of CS solution containing diclofenac sodium was 
added gradually into an equal volume of $\mathrm{CH}$ solution under the continuous magnetic stirring $(600 \mathrm{rpm})$ at room temperature and continue stirring for $15 \mathrm{~min}$ to stabilize the NPs.

\section{Characterization of developed formulation}

Particle size, size distribution, and zeta potential:

Freeze dried formulation was dispersed in ultrapure water $(1 \mathrm{mg} / 2 \mathrm{~mL})$, mean particles' size and zeta potential were determined by dynamic light scattering method using Malvern Zetasizer (Zetasizer, HAS 3000; Malvern instrument, Malvern, Worcestershire, UK). All the measurements were conducted in triplicate at a scattering angle of $90^{\circ} \mathrm{C}$ and $25^{\circ} \mathrm{C}$ temperature.

\section{Encapsulation efficiency:}

The entrapment efficiency (EE) of CS-CHNPs was evaluated by a previously reported method. ${ }^{21}$ using ultracentrifuge filtration tubes equipped with semipermeable membrane. The sample was diluted and placed in the upper chamber, followed by centrifugation for $15 \mathrm{~min}$ at $3000 \mathrm{rpm}$. The filtrate was analyzed at absorption maxima $\left(\lambda_{\max }\right) 340 \mathrm{~nm}$ by using UV-VIS, spectrophotometer (double beam, Shimadzu, Tokyo, Japan) and entrapment efficiency calculated by the following equation:

$E E(\%)=\frac{\text { Total amount of diclofenac }- \text { unentrapped diclofenac }}{\text { Total amount of diclofenac }} \times 100$

\section{In vitro release analysis:}

The developed formulation was evaluated for in-vitro release profile by dialysis bag method. ${ }^{22}$ Formulation (equivalent to $1 \mathrm{mg}$ of diclofenac) was suspended in $2 \mathrm{~mL}$ of simulated tears fluids (STF), placed into a dialysis bag; both the ends of the bag were tied and suspended into $20 \mathrm{~mL}$ of STF. Release study was performed at $32 \pm 0$. $5^{\circ} \mathrm{C}$ with stirring speed $(25 \mathrm{rpm})$. Three milliliters of samples were regularly withdrawn and analyzed at $340 \mathrm{~nm}$ by using UV-VIS spectrophotometer. Withdrawn volume was replaced with fresh media to maintain sink condition. A similar procedure was followed for marketed eye drops. All measurements were conducted in triplicate.

\section{Statistical Analysis}

All experiments were carried out in triplicate. The values were represented as mean \pm standard deviation. The differences in release profile of new developed nanoparticles formulation and marketed conventional eye drop were tested for significance by using independent two sample t-test with the aid of the statistical package for the social sciences (version 18) program. Differences were considered as statistically significant when $P<0.05$.

\section{Results}

Effect of chitosan concentration (CH)

Table 1 epitomize the effect the of chitosan concentration on the nanoparticles' size, drug entrapment, and zeta potential keeping the drug concentration $(0.1 \% \mathrm{w} / \mathrm{v})$ and chondroitin sulfate concentration $(0.1 \% \mathrm{w} / \mathrm{v})$ constant. To study the effect of chitosan concentration on nanoparticles, its concentration was taken in the range of $0.1 \%$ to $0.5 \%$. As shown in Table 1 , aggregation was observed at the lowest concentration of $\mathrm{CH} 0.1 \%$ (Formulation 1) while nanoparticles formation was taken place when the concentration of chitosan range between $0.2 \%$ to $0.5 \% \quad \mathrm{w} / \mathrm{v}$. Nanoparticles size was found to increase from $266.06 \pm 17.66 \mathrm{~nm}$ to $404.8 \pm 26.12 \mathrm{~nm}$ upon increasing chitosan concentration. It was clear that with increasing chitosan concentration, the drug entrapment was found to reduce and vice versa, while zeta potential was found to increase upon increasing chitosan concentration.

\section{Effect of chondroitin sulfate concentration (CS)}

Table 2 epitomize the effect the of chondroitin sulfate concentration on the nanoparticles' size, drug entrapment and zeta potential keeping the drug concentration $(0.1 \% \mathrm{w} / \mathrm{v})$ and chitosan concentration $(0.2 \% \mathrm{w} / \mathrm{v})$ constant. To study the effect of CS concentration, its 
concentration was taken in the range of $0.05 \%$ to $0.1 \% \mathrm{w} / \mathrm{v}$. Initially, the size of nanoparticle was found to decrease upon increasing CS, followed by an increase while drug entrapment was found to increase with increasing CS then reduce. Regarding the zeta potential, Continuous reduction in zeta potential was observed upon increasing CS. To confirm the influence of CS concentration on the particles size, drug entrapment, and zeta potential, three more formulations were also developed keeping chitosan concentration $0.15 \% \mathrm{w} / \mathrm{v}$ which revealed the similar influence as observed when $\mathrm{CH}$ was $0.2 \% \mathrm{w} / \mathrm{v}$.

Table 1: Effect of chitosan concentration on parameters (mean particles size, entrapment efficiency.

\begin{tabular}{cccccc}
\hline CH $(\% \mathrm{w} / \mathrm{v})$ & $\mathrm{CS}(\% \mathrm{w} / \mathrm{v})$ & Drug (\%w/v) & $\begin{array}{c}\text { Mean size } \\
(\mathrm{nm})\end{array}$ & EE $(\%)$ & $\begin{array}{c}\text { Zeta potential } \\
(\mathrm{mV})\end{array}$ \\
\hline 0.1 & 0.1 & 0.1 & Aggregation & - & - \\
0.2 & 0.1 & 0.1 & $266.06 \pm 17.66$ & $57.23 \pm 4.2$ & $37.11 \pm 3.74$ \\
0.3 & 0.1 & 0.1 & $315.33 \pm 21.22$ & $52.56 \pm 3.81$ & $43.63 \pm 4.12$ \\
0.4 & 0.1 & 0.1 & $386.3 \pm 24.12$ & $48.53 \pm 3.36$ & $48.55 \pm 4.52$ \\
0.5 & 0.1 & 0.1 & $404.8 \pm 26.12$ & $44.43 \pm 3.09$ & $50.65 \pm 4.74$ \\
\hline
\end{tabular}

Table 2: Effect of chondroitin sulfate concentration on parameters (mean particles size, entrapment.

\begin{tabular}{cccccc}
\hline CS (\%w/v) & CH (\%w/v) & Drug (\%w/v) & $\begin{array}{c}\text { Mean size } \\
(\mathrm{nm})\end{array}$ & EE (\%) & $\begin{array}{c}\text { Zeta potential } \\
(\mathrm{mV})\end{array}$ \\
\hline 0.05 & 0.2 & 0.1 & $337.04 \pm 24.35$ & $50.79 \pm 3.66$ & $46.28 \pm 5.55$ \\
0.075 & 0.2 & 0.1 & $232.62 \pm 11.65$ & $64.4 \pm 5.22$ & $41.99 \pm 5.02$ \\
0.1 & 0.2 & 0.1 & $266.06 \pm 17.66$ & $57.23 \pm 4.2$ & $37.11 \pm 4.13$ \\
0.05 & 0.15 & 0.1 & $263.6 \pm 17.22$ & $55.86 \pm 4.36$ & $38.62 \pm 5.52$ \\
0.075 & 0.15 & 0.1 & $220.15 \pm 10.89$ & $69.03 \pm 5.46$ & $36.44 \pm 4.69$ \\
0.1 & 0.15 & 0.1 & $239.8 \pm 12.85$ & $61.36 \pm 4.82$ & $28.82 \pm 4.32$ \\
\hline
\end{tabular}




\section{Effect of drug concentration}

Table 3 epitomize the effect the of drug concentration on the nanoparticles' size, drug entrapment, and zeta potential while keeping CS concentration $(0.075 \% \mathrm{w} / \mathrm{v})$ and $\mathrm{CH}$ concentration $(0.2 \% \mathrm{w} / \mathrm{v})$ constant. To study the effect of diclofenac sodium, its concentration was taken in the range of $0.05 \%$ to $0.2 \% \mathrm{w} / \mathrm{v}$. As summarized in Table 3, the drug concentration directly influenced the particles size, i.e., while increasing the concentration of the drug, the particles size was found to increase.
The same increment was observed on the entrapment efficacy while zeta potential decrease upon increasing concentration of diclofenac sodium. To confirm the influence of initial drug concentration on the particles size, drug entrapment, and zeta potential, three more formulations were also developed keeping chitosan concentration $0.15 \% \mathrm{w} / \mathrm{v}$ which revealed the similar influence as observed when chitosan concentration was $0.2 \% \mathrm{w} / \mathrm{v}$. No nanoparticles were observed when the concentration of drug was $0.2 \% \mathrm{w} / \mathrm{v}$.

Table 3: Effect of initial drug concentration on three parameters (mean particles size, entrapment.

\begin{tabular}{|c|c|c|c|c|c|}
\hline Drug (\%w/v) & $\mathrm{CH}(\% w / v)$ & CS (\%w/v) & $\begin{array}{l}\text { Mean size } \\
\quad(\mathrm{nm})\end{array}$ & EE (\%) & $\begin{array}{l}\text { Zeta potential } \\
(\mathrm{mV})\end{array}$ \\
\hline 0.05 & 0.2 & 0.075 & $204.82 \pm 11.32$ & $48.92 \pm 3.21$ & $44.95 \pm 6.16$ \\
\hline 0.1 & 0.2 & 0.075 & $232.62 \pm 11.65$ & $64.4 \pm 5.22$ & $41.99 \pm 5.02$ \\
\hline 0.15 & 0.2 & 0.075 & $251.1 \pm 15.12$ & $73.02 \pm 5.33$ & $38.89 \pm 5.05$ \\
\hline 0.2 & 0.2 & 0.075 & Aggregation & - & - \\
\hline 0.05 & 0.15 & 0.075 & $180.3 \pm 11.65$ & $50.93 \pm 3.33$ & $39.76 \pm 5.02$ \\
\hline 0.1 & 0.15 & 0.075 & $220.15 \pm 10.89$ & $69.03 \pm 5.46$ & $36.44 \pm 4.69$ \\
\hline 0.15 & 0.15 & 0.075 & $332.5 \pm 22.64$ & $73.42 \pm 6.22$ & $32.53 \pm 3.77$ \\
\hline 0.2 & 0.15 & 0.075 & Aggregation & - & - \\
\hline
\end{tabular}




\section{In vitro release profile}

The release pattern of developed nanoparticles and conventional eye drops were compared, and the results are shown in Figure 1 and 2. As expected, the conventional eye drops shows immediate release profile of approximately more than $50 \%$ of the drug within 30 minutes while only $15 \pm 1.13 \%$ of drug released from the developed nanoparticle within one hour, the release of drug from conventional eye drops and developed nanoparticles was found to be $90.1 \pm 1.09 \%$ and $25.6 \pm 1.82 \%$, respectively. A significant difference in the release of drug from both formulas was explained at the end of 3 hours of the experiment $(P=0.018)$ in which $98.2 \pm 1.13 \%$ of drug released from marketed eye drop and $38.9 \pm 0.65 \%$ of drug released from the CS-CH-NPs. Also, CS-CH-NPs showed sustained release profile $(95.7 \pm 0.99 \%)$ within 24 hours, as shown in Figure 1.

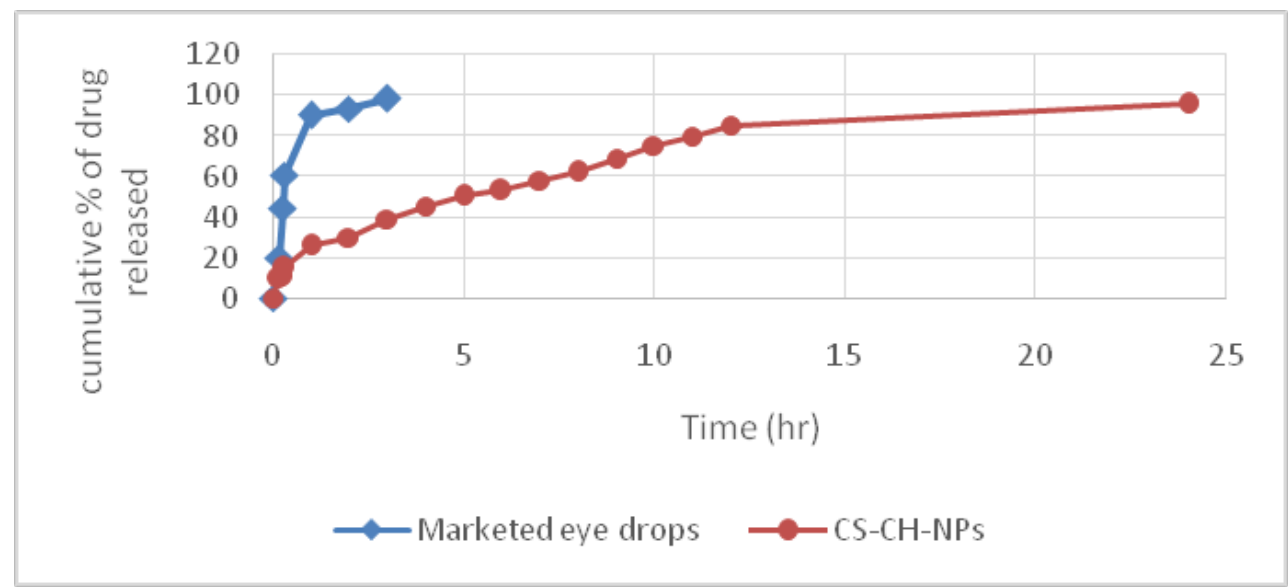

Figure 1: In vitro release profile of diclofenac sodium from the marketed eye drops and CS-CH-NPs.

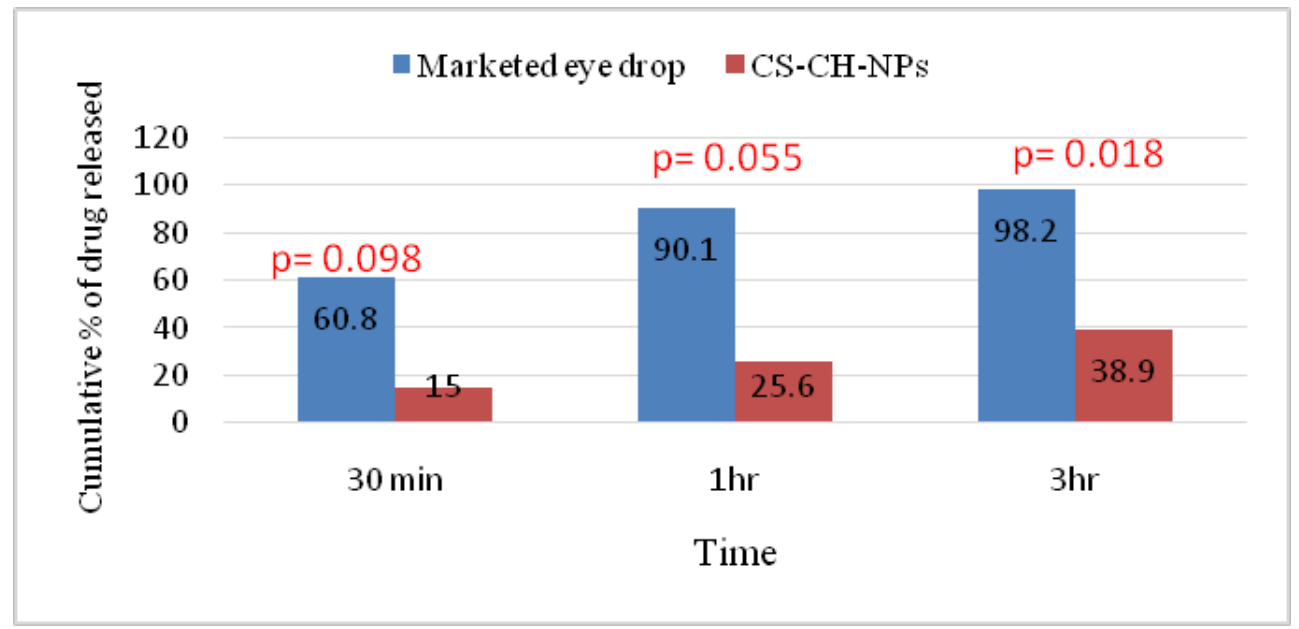

Figure 2: Comparison in the release profile of diclofenac sodium from marketed eye drops and $\mathrm{CS}-\mathrm{CH}-\mathrm{NPS}$ at $30 \mathrm{~min}, 1 \mathrm{hr}$, and $3 \mathrm{hr}$. 


\section{Discussion}

For the development and optimization of diclofenac sodium loaded CS-CH-NPs, we depended on ionic interaction between cationic amino group of $\mathrm{CHs}$ and anionic sulfate group of CS as it results in gelation and thus the formation of nanoparticles aggregates. It may induce clear solution, opalescent suspensions, and aggregation depending on the concentration of $\mathrm{CS}, \mathrm{CH}$, and drugs; however, the area of opalescent is considered to be the most appropriate for the development of NPs. Furthermore, $\mathrm{pH}$ affects the particles' size of the formulations, so the $\mathrm{pH}$ of $\mathrm{CH}$ solution was kept at 5 as particles size and maximum yield were reported at this $\mathrm{pH}^{23}$ Three parameters (particles' size, entrapment efficiency, and zeta potential) were used to study the effect of concentration of $\mathrm{CH}$, CS, and drug on formulations.

\section{Effect of chitosan concentration:}

The effect of chitosan concentration on the particle's size, drug entrapment, and zeta potential summarizes in Table 1. The NPs formation took place at the concentration range of $0.2-0.5 \% \mathrm{w} / \mathrm{v}$ while aggregation was observed at the lowest concentration of $\mathrm{CH}(0.1 \% \mathrm{w} / \mathrm{v})$. The NPs formation occurs due to ionic interaction between cationic molecules of $\mathrm{CH}$ and anionic molecules of CS, but this formation of NPS took place at a specific weight ratio of cationic and anionic polyelectrolytes. ${ }^{24}$ Thus, aggregation at concentration $0.1 \%$ $w / v$ of $\mathrm{CH}$ happen when the ratio of $\mathrm{CH}$ : CS is $(1: 1)$ because at this ratio CS would be higher than required to stabilize the particles so over aggregation of CS molecules on the NPs owing to attraction between $\mathrm{CS}$ and $\mathrm{CH}$, thus aggregate formation. With further increase in the concentration of $\mathrm{CH}$ the amount of CS remains within the required range to stabilize the particles and consequently NPs formation. While increasing in particles' size with increasing in $\mathrm{CH}$ concentration can be explained by relatively lower amount of CS (constant $0.1 \% \mathrm{w} / \mathrm{v}$ ) as the repulsive force between
$\mathrm{CH}-\mathrm{CH}$ molecules overcome the attraction force between $\mathrm{CH}-\mathrm{CS}$ molecules and because higher attraction force is required for smaller size NPs, ${ }^{25}$ so larger size NPs at a higher $\mathrm{CH}$ concentration. On the other hand, drug entrapment was found to reduce upon increasing $\mathrm{CH}$ concentration due to lose network within larger NPs because of more repulsion among $\mathrm{CH}-\mathrm{CH}$ amino group, thus more leaching of drug and poor entrapment. The availability of more number of positively charged $\mathrm{CH}$ mean more repulsion and higher zeta potential.

\section{Effect of chondroitin sulfate concentration:}

The effect of chondroitin sulfate concentration on particles' size, drug entrapment, and zeta potential summarized in Table 2. As the concentration of CS increased, the size of NPs first decreases then followed by an increment. The first reduction in particles' size happens because CS at this value was in an optimum amount to establish the optimum balance between attractive force among $\mathrm{CS}-\mathrm{CH}$ molecules and repulsive force among $\mathrm{CH}-\mathrm{CH}$ molecules, which result in more shrinkage of polymers so smaller size particle. Further increase in the concentration of CS results in larger size particle. ${ }^{26}$ The drug entrapment efficacy first was found to increase upon increasing in CS concentration then decreased with further increment in CS concentration. The first increment was explained by the fact that at this amount of CS the size of NPs was smaller so denser network but when the amount of CS was higher, the size of NPs was larger. Consequently, there is a loose network and more leaching of the drug. The concentration of the CS effect on Zeta potential as a continuous reduction in zeta potential was observed upon the increasing amount of CS. The feasible reason for the reduction in zeta potential could be more neutralization of cationic groups of $\mathrm{CH}$ molecules with anionic CS molecule, so the reduction in positive zeta 
potential. To further verify the role of CS concentration on prepared NPs, further formulas were prepared keeping the concentration of $\mathrm{CH} 0.15 \% \mathrm{w} / \mathrm{v}$ constant. The same observation for particles' size, drug entrapment, and zeta potential were obtained. However, only the point that we observed from the change was that the effects were found to be higher than observed at $0.2 \% \mathrm{w} / \mathrm{v} \mathrm{CH}$ due to the loose aggregation of excess CS molecules on the NPs because of the dominance of repulsive force over attractive force so larger size particle.

\section{Effect of drug concentration}

Effect of initial drug concentration on the nanoparticles size, their drug entrapment, and zeta potential were summarized in Table 3. The results showed an increase in the size of nanoparticles upon increasing the initial drug concentration. This can be explained by two facts. First, since diclofenac sodium is an anionic drug when entrapped into the particles, it enhances the repulsive force induced by CS-CS molecules, i.e., synergized the repulsive force. Second, diclofenac molecules competitively inhibit the interaction between molecules of $\mathrm{CH}-\mathrm{CS}$, which is responsible for shrinking particles so as the drug concentration increased the discussed reasons become more prominent. ${ }^{26}$ There was a direct relationship between initial drug concentration and entrapment efficacy; it can be explained by an effective collision between drug molecules and $\mathrm{CH}$ molecules. As the number of reactant molecules increased, the effective collision was increased, i.e., as the concentration of drug increased, more reaction between drug molecules and $\mathrm{CH}$ molecules would happen (more entrapment of drug within particles). ${ }^{27}$ Entrapment of anionic drug molecules within particles leads to neutralize the positive charge of $\mathrm{CH}$ molecules and subsequently, a reduction in zeta potential values.

In vitro release profile

The effect of different variables such as
$\mathrm{CH}, \mathrm{CS}$, and initial drug concentration on three parameters (particles' size, drug entrapment, and zeta potential) were studied and summarized in Table 1-3. Depending on the results, the optimized formulation was selected. The optimized formula showed the particles size $251.1 \pm 15.12 \mathrm{~nm}$, entrapment efficacy $73.02 \pm 5.33 \%$, and zeta potential $38.89 \pm 5.05 \mathrm{mV}$. In vitro release profile of the optimized formula was carried out and compared with the release profile of marketed eye drops. The developed formula showed biphasic release pattern; initial burst release was about $25.6 \pm 1.82 \%$ in $1 \mathrm{hrs}$ followed by sustained release of about $95.7 \pm 6.36 \%$ within $24 \mathrm{hrs}$. The initial burst release was due to rapid hydration of NPs, resulted in leaching of loosely bound/ surface adhered drug molecules, but the sustained release due to the swelling property of $\mathrm{CH}$, which act as a matrix then the entrapped drug within the swelled matrix, diffuses out gradually. A significant difference in release profile of developed formula CS-CH-NPs and the marketed conventional eye drops was obtained. The conventional eye drops showed an immediate release of more than $50 \%$ within 30 minutes. Biphasic release of developed formula seen as an advantage toward improved delivery.

\section{Conclusion}

Diclofenac loaded CS-CH-NPs were successfully developed depending on three parameters CS concentration, $\mathrm{CH}$ concentration, and initial drug concentration. The selected formula demonstrated particles size in Nanodimension with good entrapment of diclofenac. In vitro release profile of the new formula demonstrated biphasic release pattern in which initial burst release provide a rapid effect of drug followed by a sustained release pattern for about 24 hours. The developed nanoparticles could be a substitute for improved ocular delivery of diclofenac. 


\section{Competing interests}

The authors declare that they have no competing interests.

\section{References}

1. Patel A, Cholkar K, Agrahari V, Mitra AK. Ocular drug delivery systems: An overview. World J Pharmacol 2013; 2:47-64.

2. Teodora I, Mihaela VG, Lacramioara P, Valentina A, Andreea-Letitia A, Cristina-Elena D. Strategies for improving ocular drug bio availability and corneal wound healing with chitosan-based delivery systems. Polymers 2018; 10:221.

3. Baranowski P, Karolewicz B, Gajda M, Pluta J. Ophthalmic Drug Dosage Forms: Characterisation and Research Methods. Sci. World J 2014; 2014:1-14.

4. Rupenthal ID. Ocular Drug Delivery Technologies: Exciting Times Ahead. ON drug Delivery 2015; 54:7-11.

5. Anita $U$, Lidia $T$. Design of chondroitin sulfate- based polyelectrolyte nanoplexes: Formation of nanocarriers with chitosan and a case study of salmon calcitonin. Carbohydr Polym 2017; 156:276-84.

6. Ali M. Nanocarriers of nanotechnology in retinal diseases. Saudi J Ophthalmol 2014; 28:304-9.

7. Guinedi AS, Mortada ND, Mansour S, Hathout RM. Preparationand evaluation of reverse-phase evaporation and multilamellarniosomes as ophthalmic carriers of acetazolamide. Int J Pharm 2005; 306:71-82.

8. Gupta H, Jain S, Mathur R, Mishra P, Mishra AK, Velpandian T. Sustained ocular drug delivery from a temperature and $\mathrm{pH}$ triggered novel in situ gel system. Drug Deliv 2007; 14:507-15.

9. De la Fuente M, Ravina M, Paolicelli $P$, Sanchez A, Seijo B, Alonso MJ. Chitosan-based nanostructures: a delivery platform for ocular therapeutic chitosan [J]. Adv Drug Deliv Rev 2010; 62(1):100-17.

10. Mun EA, Morrison PWJ, Williams AC, Khutoryanskiy VV. On the barrier properties of the cornea: a microscopy study of the penetration of fluorescently labeled nanoparticles, polymers, and sodium fluorescein [J]. Mol Pharm 2014; 11(10):3556-64.

11. Chen WB, Wang LF, Chen JS, Fan SY. Characterization of polyelectrolyte complexes between chondroitin sulfate and chitosan in the solid state. J Biomed Mater Res 2005; 75(1):128-37.

12. Kouchak M, Avadi M, Abbaspour M, Jahangiri A, Kargar Boldaji S. Effect of different molecular weights of chitosan on preparation and characterization of insulin loaded nanoparticles by ion gelation method. Int J Drug Dev Res 2012; 4(2):271-7.

13. Vicekjot B, Gurpreet K. Preparation and characterization of polyelectrolyte complexes of Hibiscus esculentus (Okra) gum and chitosan.
Int J Biomater 2018; 2018:4856287.

14. Thunemann AF, Müller $M$, Dautzenberg $H$, Joanny J, Löwen H. Polyelectrolytes with Defined Molecular Architecture II. Vol. 166. Berlin, Heidelberg: Springer Berlin Heidelberg: Polyelectrolyte Complexes; 2004. P. 113-71 (Advances in Polymer Science).

15. Araújo J, Gonzalez E, Egea MA, Garcia ML, Souto EB. Nanomedicines for ocular NSAIDs: safety on drug delivery. Nanomedicine 2009; 5:394-401.

16. Ahuja M, Sharma SK, Majumdar DK. In vitro corneal permeation of diclofenac from oil drops. Yakugaku Zasshi 2007; 127:1739-45.

17. Attama AA, Reichl S, Müller-Goymann CC. Diclofenac sodium delivery to the eye: in vitro evaluation of novel solid lipid nanoparticle formulation using human cornea construct. Int J Pharm 2008; 355:307-13.

18. Agnihotri SM, Vavia PR. Diclofenac-loaded bio-polymericnano-suspensions for ophthalmic application. Nanomedicine 2009; 5:90-5.

19. Yosra A, Jiaxin L, Sujay P, Ali S, Ilva D. Nanoparticle cross-linked collagen shields for sustained delivery of pilocarpine hydrochloride. Int J Pharm 2016; 501:96-101.

20. Kirandeep K, Gurpreet K. Formulation and evaluation of chitosan- chondroitin sulfate based nasal inserts for zolmitriptan. BioMed Res Int 2013; 2013:958465.

21. Chander PD, Shailendra KS, Sanjeev K, Ashok KD, Aakash D. Development and characterization of nanoparticles of Glibenclamide by solvent displacement method. Acta Poloniae Pharmaceutica- drug Research 2010; 67(2):283-90.

22. Kaskoos RA. Investigation of moxifloxacin loaded chitosan-dextran nanoparticles for topical instillation into eye: In-vitro and ex- vivo evaluation. Int J Pharm Investg 2014;4:167-73.

23. Aydin RS, Mehlika Pulat. 5-Fluorouracil encapsulated chitosan nanoparticles for $\mathrm{pH}$ stimulated drug delivery: Evaluation of controlled release kinetics. J Nanomater 2012; 2012:313961.

24. Usha G, Sree H, Balakeshwa R, Prakash R. Improved intraocular bioavailability of ganciclovir by mucoadhesive polymer based ocular microspheres: development and simulation process in Wister rats. Daru Journal of Pharmaceutical Sciences 2015; 23(1):49.

25. Ameeduzzafar, Ali J, Bhatnagar A, Kumar N, Ali A. Chitosan nanoparticles amplify the ocular hypotensive effect of cateolol in rabbits. Int J Biol Macromol 2014; 65:479-91.

26. Javier $P Q$, Hazel $P$, Carlos $P$. Chitosan based self-assembled nanoparticles in drug delivery. Polymers 2018; 10(3):235.

27. Megha A, Negar DP, Nalini S, Agarwal MK. Chitosan nanoparticles based drug delivery: An update. IJAMR 2015; 2(4):1-13. 